\title{
A Quantum Dynamics Study of the Ultrafast Relaxation in a Prototypical $\mathrm{Cu}(\mathrm{I})$-Phenanthroline
}

\author{
G. Capano, ${ }^{\dagger, \ddagger}$ M. Chergui, ${ }^{\dagger}$ U. Rothlisberger, ${ }^{\ddagger}$ I. Tavernelli, ${ }^{\ddagger}$ and T. J. Penfold ${ }^{*}, \S$ \\ ${ }^{\dagger}$ Laboratoire de spectroscopie ultrarapide, ISIC, École Polytechnique Fédérale de Lausanne (EPFL), FSB Station 6, CH-1015 \\ Lausanne, Switzerland \\ ${ }^{\ddagger}$ Laboratoire de chimie et biochimie computationnelles, ISIC, FSB-BCH, École Polytechnique Fédérale de Lausanne (EPFL), \\ CH-1015 Lausanne, Switzerland \\ ${ }^{\S}$ SwissFEL, Paul Scherrer Institute, CH-5232 Villigen, Switzerland
}

Supporting Information

ABSTRACT: The ultrafast nonadiabatic dynamics of a prototypical $\mathrm{Cu}(\mathrm{I})-$ phenanthroline complex, $\left[\mathrm{Cu}(\mathrm{dmp})_{2}\right]^{+}(\mathrm{dmp}=2$,9-dimethyl-1,10-phenanthroline), initiated after photoexcitation into the optically bright metal-toligand charge-transfer (MLCT) state $\left(\mathrm{S}_{3}\right)$ is investigated using quantum nuclear dynamics. In agreement with recent experimental conclusions, we find that the system undergoes rapid $(\sim 100 \mathrm{fs})$ internal conversion from $S_{3}$ into the $S_{2}$ and $S_{1}$ states at or near the Franck-Condon (FC) geometry. This is preceded by a dynamic component with a time constant of $\sim 400 \mathrm{fs}$, which corresponds to the flattening of the ligands associated with the pseudo JahnTeller distortion. Importantly, our simulations demonstrate that this latter aspect is in competition with subpicosecond intersystem crossing (ISC). The mechanism for ISC is shown to be a dynamic effect, in the sense that it arises from the system traversing the pseudo Jahn-Teller coordinate where the singlet and triplet states become degenerate, leading to efficient crossing. These first-principles quantum dynamics simulations, in conjunction with recent experiments, allow us to clearly resolve the mechanistic details of the ultrafast dynamics within $\left[\mathrm{Cu}(\mathrm{dmp})_{2}\right]^{+}$, which have been disputed in the literature.

\section{INTRODUCTION}

Owing to their strong absorption in the UV-visible region of the spectrum and to their long-lived excited states, transition metal complexes have become a central component of photocatalysts, ${ }^{1}$ dye-sensitized solar cells (DSSCs), ${ }^{2}$ and organic light emitting diodes (OLEDs). ${ }^{3}$ Their extensive use for such applications has been the main driving force for a large research effort aimed at understanding their fundamental photophysical and photochemical properties. Many of these studies have focused upon resolving the decay mechanisms and the ultimate fate of the initially excited state, which generally occurs on the femtosecond time scale and can often be important for describing the suitability and efficiency of a complex for a particular application.

For a complete understanding of these ultrafast processes, simulations are an important tool. Computationally, the most common approach to address such problems is by calculating energy profiles along viable reaction and/or decay pathways. While useful, the resulting picture is static and lacks the dynamical information important for obtaining a complete understanding. In particular, because excited state dynamics are characterized by the breakdown of the Born-Oppenheimer approximation, ${ }^{4,5}$ these simulations must not only describe the profile of the excited state surfaces but also accurately incorporate the effects of the coupling between the different states that lead to multiple nonadiabatic relaxation channels.

These nonadiabatic couplings give rise to the internal conversion (IC). However, for transitions metal complexes it is often important to also consider the intersystem crossing (ISC) relaxation processes, ${ }^{6}$ which can be decisive for understanding both the decay mechanisms and light-harvesting efficiency. ${ }^{7}$ The former (IC) involves population transfer between states of the same spin multiplicity, whereas the latter arises from the spin-orbit coupling (SOC) interaction between states of different multiplicity. ${ }^{8}$ Following ultrafast ISC reported for prototypical transition metal complexes, such as $[\mathrm{Ru}-$ (bpy) $\left.]_{3}\right]^{2+9-12}$ it is often assumed that the ISC rate occurs on the subpicosecond time scale and scales with the heavy atom effect. However, notable exceptions ${ }^{13-15}$ highlight that this is not a general rule and that the rate of ISC is determined not only by the presence of heavy elements but also by the molecular structure and the nature of the electronic states involved. These can only be accurately assessed by calculating the magnitude of the SOC matrix elements at important points

Received: September 25, 2014

Revised: October 2, 2014

Published: October 2, 2014 
along the potential energy surface ${ }^{16,17}$ and following the time evolution of the system.

From this perspective a class of systems whose ultrafast dynamics and ISC rate have received significant attention are the $\mathrm{Cu}(\mathrm{I})$-phenanthroline complexes, ${ }^{18,19}$ among which $[\mathrm{Cu}-$ $\left.(\mathrm{dmp})_{2}\right]^{+}$is a prototypical example. These complexes have been studied for potential application as photosensitizers and in this regard have many properties similar to those of the ruthenium polypyridines. ${ }^{20}$ However, they exhibit a lower coordination number of 4 , which though offering greater flexibility to fine-tune their photophysical properties, also leads to strong structure-dependent energetics and a susceptibility to solvent effects. In general, the ground state of these complexes adopts a pseudotetrahedral geometry with the two ligands being orthogonal. ${ }^{21}$ Upon excitation into the singlet metal-toligand-charge-transfer (MLCT) states the oxidation state of copper becomes $\mathrm{Cu}$ (II) and a flattening of the complex occurs (Figure 1), because the copper $\mathrm{d}^{9}$ electronic configuration is

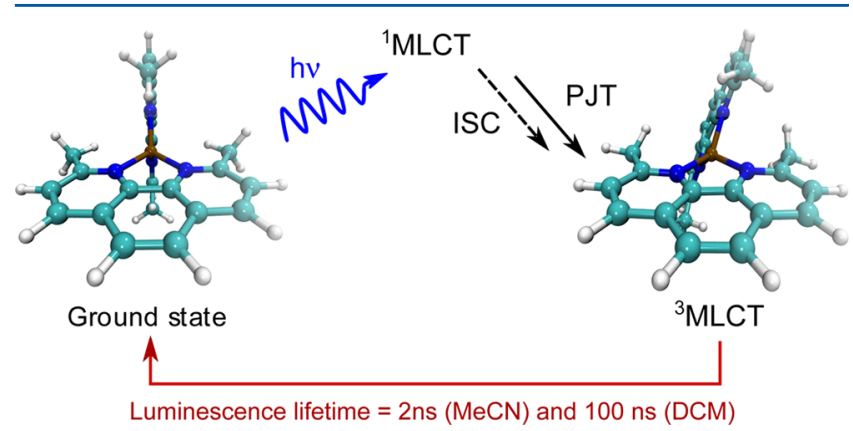

Figure 1. General schematic of the excited state dynamics of $\left[\mathrm{Cu}(\mathrm{dmp})_{2}\right]^{+} . \mathrm{MeCN}=$ acetonitrile and $\mathrm{DCM}=$ dichloromethane. See main text for details.

susceptible to pseudo Jahn-Teller (PJT) distortions. On the time scale of tens of picoseconds, the system is relaxed into a long-lived triplet state; however, the lifetime of this state is strongly solvent dependent. ${ }^{18,22-24}$ To overcome the influence of the surrounding solvent, and prolong the excited state lifetime, bulky side chains have been added to the phenanthroline ligands. ${ }^{19,25,26}$ However, these affect not only the longer time dynamics associated with the solvent effect but also the femtosecond dynamics. ${ }^{27}$

One of the first time-resolved spectroscopic studies of $\left[\mathrm{Cu}(\mathrm{dmp})_{2}\right]^{+}$was performed by Chen et al., ${ }^{28}$ who used timeresolved absorption spectroscopy to probe the dynamics following photoexcitation of the optically bright $S_{3}$ state. $^{29}$ They reported two principal dynamical processes occurring in $500-700 \mathrm{fs}$ and $10-20$ ps. On the basis of the ultrafast ISC occurring in $\left[\mathrm{Ru}(\mathrm{bpy})_{3}\right]^{2+}$, they assigned the first to ultrafast ISC and the second to the structural relaxation, i.e., the PJT distortion. At the same time Nozaki and co-workers ${ }^{30}$ reported that the $10-20$ ps relaxation was due to ISC, which is relatively slow in $\left[\mathrm{Cu}(\mathrm{dmp})_{2}\right]^{+}$due to a strong effect of the structural rearrangements on the magnitude of the SOC matrix elements. Indeed, at the FC geometry the coupling between $S_{1}$ and $T_{1}$ was estimated to be $\sim 300 \mathrm{~cm}^{-1}$, but at the distorted excited state geometry, expected to be most important on the time scale of tens of picosecond, it is only $\sim 30 \mathrm{~cm}^{-1}$. However, the authors also concluded that the quantum yield of the lowest singlet state in the PJT relaxed geometry $\left(S_{1}^{\mathrm{PJT}}\right)$ was $\sim 0.1$ and suggested that this pathway competes with the ultrafast ISC to the ${ }^{3}$ MLCT occurring at the FC geometry, which is therefore the dominant relaxation channel.

Later, Tahara and co-workers ${ }^{32}$ probed the excited state dynamics using femtosecond fluorescence up-conversion. Following photoexcitation at $420 \mathrm{~nm}$ they found three principal time components. An initial strong fluorescence, which decayed with a time constant of $\sim 45 \mathrm{fs}$, was attributed to the decay of the initially populated excited state via IC. This was followed by two time components of $660 \mathrm{fs}$ and $7.4 \mathrm{ps}$, assigned to the PJT distortion and ISC, respectively. Later Chen and co-workers ${ }^{33}$ reported similar time components, $80 \mathrm{fs}, 510 \mathrm{fs}$, and $10-15$ ps. They concluded, in agreement with Tahara, that the longer time scale was ISC and assigned the shortest time constant ( 80 fs) to IC and the PJT distortion. Finally, Tahara and coworkers ${ }^{34}$ used transient absorption spectroscopy to investigate the dynamics following excitation at $550 \mathrm{~nm}$. This transition corresponds to the lowest singlet MLCT state $\left(S_{1}\right)$, which is dipole forbidden but gains intensity through vibronic coupling with the main absorption band. They observed distinct wavepacket dynamics corresponding to the PJT distortion and were able to obtain a low-frequency vibrational spectrum of the important modes. As the damping time for the oscillations were similar to that of the structural change, the authors concluded that the wavepacket dynamics arises from the coherent nuclear motion of the $S_{1}$ state before the structural change. They proposed the presence of a small but finite potential barrier between the perpendicular and flattened $S_{1}$ states, which using time-dependent density functional theory (TDDFT) was found to have a height of $0.002 \mathrm{eV} .{ }^{34}$ However, this is at odds with the spontaneous structural instability usually associated with PJT effects and the reported barrier is both well below the accuracy of TDDFT and an order of magnitude smaller than $k T$ at $300 \mathrm{~K}$

All of these latter studies ${ }^{27,32-34}$ rule out ultrafast ISC. However, the analysis of these experiments only considered SOC between the $S_{1}$ and $T_{1}$ states. As pointed out by Zgierski, $^{21}$ there are four closely spaced triplet states in close proximity to $S_{1}$, and therefore the $S_{1}-T_{1}$ coupling is not the only relaxation channel into the triplet states. Indeed, it was shown in ref 31 that along a PJT $\left(\nu_{21}\right)$ mode identified to be important in the wavepacket dynamics of ref 34 the $T_{2}, T_{3}$, and $\mathrm{T}_{4}$ states all intersect the $\mathrm{S}_{1}$ state (Figure $2 \mathrm{~b}$ ). Therefore, one could expect that efficient ISC could occur via a dynamic effect in which the system traverses a region where coupled states are degenerate leading to a more efficient crossing. ${ }^{35}$

Consequently, it is apparent that a complete and consistent rationalization of the initial dynamics reported by Chen et al. $^{28,33}$ and Tahara et al. ${ }^{32}$ is still missing. To shed light on these dynamics and provide a complete picture of the photophysical processes, we present a study of the femtosecond excited state dynamics of $\left[\mathrm{Cu}(\mathrm{dmp})_{2}\right]^{+}$, using quantum nuclear wavepacket dynamics within the framework of the multiconfigurationaltime-dependent-Hartree (MCTDH) method. ${ }^{36}$ We show that IC from the initially photoexcited state to the lowest lying singlet state occurs in $\sim 100$ fs followed by the pseudo-JahnTeller distortion in $\sim 400 \mathrm{fs}$. These time scales agrees well with the dynamics previously reported. ${ }^{28,32,33}$ By calculating the SOC matrix elements between all of the close lying states we find that $\sim 80 \%$ of the wavepacket crosses into the triplet states within 1 ps, demonstrating that ultrafast ISC does occur in $\left[\mathrm{Cu}(\mathrm{dmp})_{2}\right]^{+}$and competes with the structural dynamics associated with the PJT distortion. 

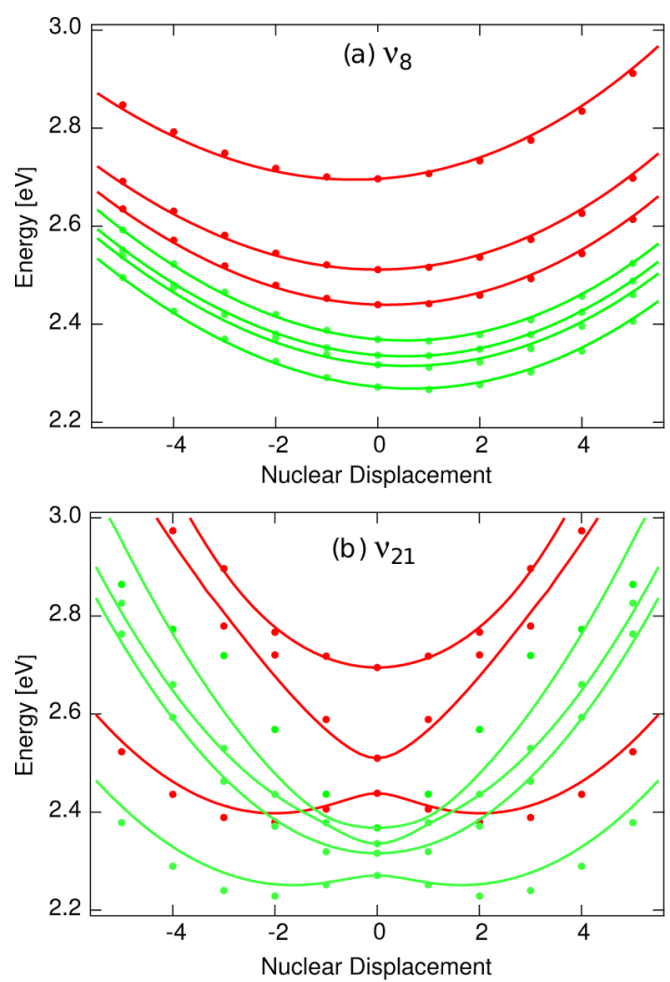

Figure 2. Cuts through the PES along (a) $\nu_{8}$ and (b) $\nu_{21}$. The dots are results from the quantum chemistry calculations for the singlet (red) and triplet (green) states. The lines correspond to their fit from which the expansion coefficients are determined.

\section{THEORY}

Vibronic Coupling Hamiltonian. The adiabatic electronic basis, provided by quantum chemistry calculations, is unsuitable for quantum dynamics simulations because the coupling vectors between electronic states states becomes infinite when two states become degenerate. These singularities can be removed by switching to a diabatic electronic basis, which is therefore the method of choice for quantum dynamics simulations. The adiabatic picture provides a sets of energy-ordered potential energy surfaces and nonlocal couplings elements via nuclear momentum-like operators. In contrast, in the diabatic picture the potential energy surface is related to an electronic configuration and the couplings, are provided by local multiplicative Q-dependent potential-like operators. Importantly, as the surfaces in the diabatic picture are smooth they can often be described by a low-order Taylor expansion.

The Hamiltonian used in the present work is based upon the vibronic coupling Hamiltonian described in ref 37 . Here the diabatic basis is fixed by choosing a point at which it is equal to the adiabatic basis (i.e., the coupling is zero), in this case the Franck-Condon point. The Hamiltonian is expanded as a Taylor series around this point $\mathbf{Q}_{0}$, using dimensionless (massfrequency scaled) normal mode coordinates:

$$
\mathbf{H}=\mathbf{H}^{(0)}+\mathbf{W}^{(0)}+\mathbf{W}^{(1)}+\ldots
$$

The zeroth-order term is the ground state harmonic oscillator approximation:

$$
H^{(0)}=\sum_{\alpha} \frac{\omega_{\alpha}}{2}\left(\frac{\partial^{2}}{\partial Q_{\alpha}{ }^{2}}+Q_{\alpha}^{2}\right)
$$

with the vibrational frequencies $\omega_{\alpha}$. The zeroth-order coupling matrix contains the adiabatic state energies at $\mathbf{Q}_{0}$. The adiabatic potential surfaces are equal to the diabatic surfaces at this point, so $\mathbf{W}^{(0)}$ is diagonal and is expressed

$$
W_{i j}^{(0)}=\sum_{\alpha}\left\langle\Phi_{i}\left(\mathbf{Q}_{0}\right)\left|H_{\mathrm{el}}\right| \Phi_{j}\left(\mathbf{Q}_{0}\right)\right\rangle
$$

where $\mathbf{H}_{\mathrm{el}}$ is the standard clamped nucleus electronic Hamiltonian and $\Phi$ are the diabatic electronic functions. The first-order linear coupling matrix elements are written

$$
W_{i j}^{(1)}=\sum_{\alpha}\left\langle\Phi_{i}\left(\mathbf{Q}_{0}\right)\left|\frac{\partial H_{\mathrm{el}}}{\partial Q_{\alpha}}\right| \Phi_{j}\left(\mathbf{Q}_{0}\right)\right\rangle Q_{\alpha}
$$

where the on-diagonal terms are expressed

$$
W_{i i}^{(1)}=\sum_{\alpha} \kappa_{\alpha}^{(i)} Q_{\alpha}
$$

and the off-diagonal

$$
W_{i j}^{(1)}=\sum_{\alpha} \lambda_{\alpha}^{(i, j)} Q_{\alpha}
$$

$\kappa$ and $\lambda$ are the expansion coefficients corresponding to the onand off- diagonal matrix elements. The on-diagonal elements are the forces acting within an electronic surface and the offdiagonal elements are the nonadiabatic couplings. Higher order terms can be important and are included in the same manner. ${ }^{38}$

The expansion coefficients for these coupling matrix elements (Tables S1-6, Supporting Information) were obtained by performing a fit to DFT/TDDFT calculations at various geometries along the important normal modes. These normal modes were calculated using DFT as implemented in Gaussian $09^{39}$ within the approximation of the M06 functional. $^{40}$ which gave good agreement with the experimental vibrational modes reported in ref 34 . The DFT/TDDFT energies were obtained within the approximation of the B3LYP functional, ${ }^{41-44}$ which gave good agreement with the experimental absorption spectrum. For all calculations a TZVP basis set was used for the copper atom and an augSVP basis set for N, C, and H. Further details are given in ref 31 and in the Supporting Information.

Importantly, as $\left[\mathrm{Cu}(\mathrm{dmp})_{2}\right]^{+}$has 57 atoms, and therefore 165 nuclear degrees of freedom, calculating the full PES is unrealistic. Consequently, to decrease the computational effort our model Hamiltonian is based upon a reduced subspace of the full configuration space, including only the electronic states in the energy range of interest and the vibrational degrees of freedom (DOF), most important for the first ps of excited state dynamics. In particular, in ref 31 we identified, using the magnitude of the linear coupling constants, 8 normal modes. Although this clearly represents a significant reduction in the dimensionality of the potential, the modes included closely correspond to those identified in the femtosecond transient absorption study of ref 34 . Consequently, although the present Hamiltonian will be unable to capture longer time effects, such as vibrational cooling, this component has little influence on the femtosecond dynamics.

To probe the role of ISC, the singlet and triplet manifolds are coupled by SOC. The SOC matrix elements at various geometries along the potential energy surface were computed with the perturbative approach developed by Wang and Ziegler $^{45}$ as implemented within ADF. ${ }^{46-48}$ A TZP basis set was used for all atoms and scalar relativistic effects were 
included using ZORA. ${ }^{49,50}$ Once calculated, the SOC matrix elements were fitted with either linear or Gaussian functions and incorporated in the Hamiltonian in the same manner as the nonadiabatic coupling terms. During the dynamics all three components $(x, y$, and $z)$ of the triplet states were treated as a single state and the SOC terms are the square root of the sum of the squares of the three couplings. The parameters and profile of the SOC matrix elements along the important modes are shown in the Tables S7 and S8 and Figures S1-S3 in the Supporting Information.

Quantum Dynamics. The quantum dynamics were performed using the Heidelberg multiconfiguration timedependent Hartree (MCTDH) package. ${ }^{36,51}$ In this approach the wave function ansatz is written as a linear combination of Hartree products:

$$
\Psi\left(Q_{1}, \ldots, Q_{f}, t\right)=\sum_{j_{1}=1}^{n_{1}} \ldots \sum_{j_{f}=1}^{n_{f}} A_{j_{1} \ldots j_{f}}(t) \prod_{k=1}^{f} \varphi_{j_{k}}^{(k)}\left(Q_{k}, t\right)
$$

where $\mathrm{Q}, \ldots, \mathrm{Q}_{f}$ are the nuclear coordinates, $A_{j_{1}, \ldots, j_{j}}(t)$ are the time-dependent expansion coefficients and $\varphi_{j_{k}}^{(k)}$ are the timedependent basis functions for each $k$ (degree of freedom), known as single particle functions (SPFs). The SPFs used in MCTDH have two advantages: (1) fewer are required as they are variationally determined and (2) the functions can be multidimensional particles containing more than one degree of freedom, thus reducing the effective number of degrees of freedom.

The dynamics were performed using two model Hamiltonians. The first (model A) includes the three lowest singlet states and the second (model B) also incorporates the four lowest triplet states. The computational details for the quantum dynamics simulations are shown in Table 1 and ensured

Table 1. Computational Details for the MCTDH Simulations of the 3-State Singlet Model (Model A) and the 7-State Intersystem Crossing Model (Model B) ${ }^{a}$

\begin{tabular}{llll} 
& modes & $N_{i}, N_{j}$ & $n_{\mathrm{S}_{1}}, n_{\mathrm{S}_{2}}, n_{\mathrm{S}_{3}}, n_{\mathrm{T}_{1}}, n_{\mathrm{T}_{2}}, n_{\mathrm{T}_{3}}, n_{\mathrm{T}_{4}}$ \\
model A & $\nu_{8}, \nu_{25}$ & 60,60 & $30,30,20$ \\
& $\nu_{19}, \nu_{21}$ & 60,60 & $30,30,20$ \\
& $\nu_{31}, \nu_{41}$ & 60,60 & $30,30,20$ \\
\multirow{3}{*}{ model B } & $\nu_{55}, \nu_{58}$ & 60,60 & $30,30,20$ \\
& $\nu_{8}, \nu_{25}$ & 60,60 & $25,25,15,25,25,15,15$ \\
& $\nu_{19}, \nu_{21}$ & 60,60 & $25,25,15,25,25,15,15$ \\
& $\nu_{31}, \nu_{41}$ & 60,60 & $25,25,15,25,25,15,15$ \\
& $\nu_{55}, \nu_{58}$ & 60,60 & $25,25,15,25,25,15,15$
\end{tabular}

${ }^{a} N_{i}, N_{j}$ are the number of primitive harmonic oscillator discrete variable representation (DVR) basis functions used to describe each mode. $n_{i}$ are the number of single-particle functions used to describe the wavepacket on each state.

convergence of the dynamics for 1 ps. For each simulation the initial wave function in the ground state, built using onedimensional harmonic oscillator functions with zero initial momentum, was vertically excited into the $S_{3}$ state at the FC geometry $(Q=0)$.

\section{RESULTS}

We now present the nonadiabatic dynamics during the first picosecond after photoexcitation. Initially, by neglecting SOC, only the dynamics within the singlet manifold are considered.
In the second subsection, the SOC matrix elements are included and their influence on ISC and its rate is assessed.

Singlet Nonadiabatic Dynamics. Figure 3a shows the relative diabatic state populations during the first picosecond
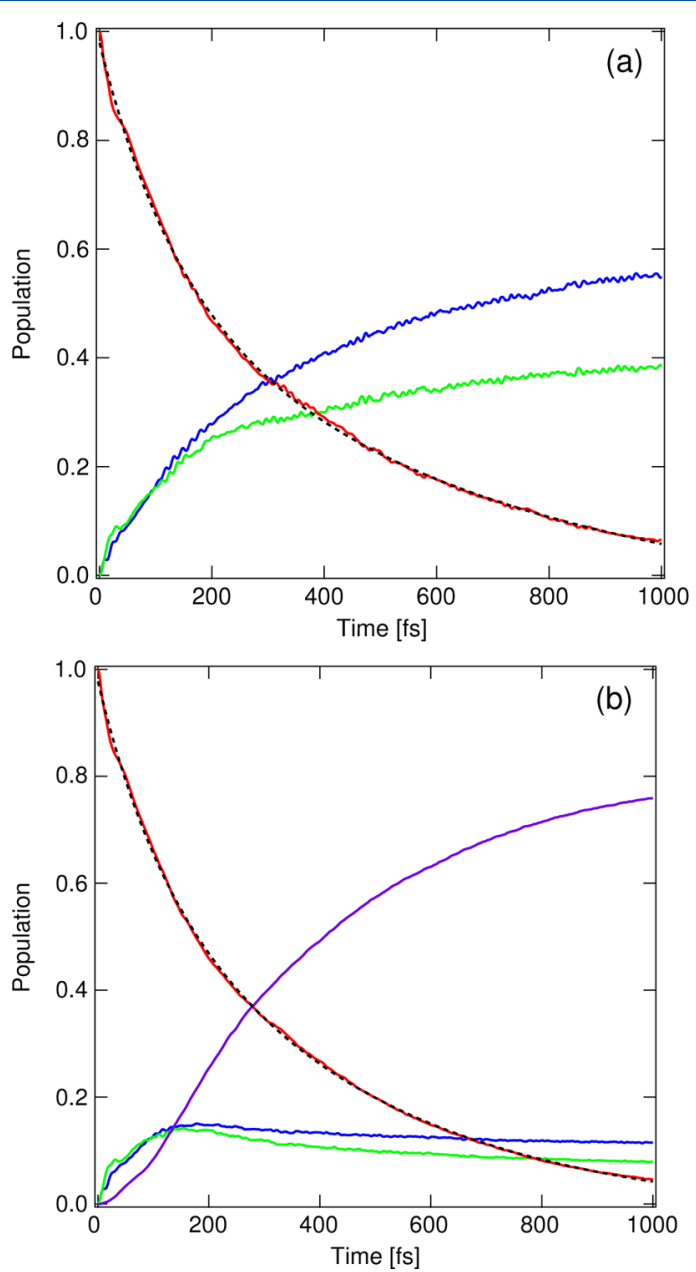

Figure 3. (a) Relative diabatic state populations of $S_{1}$ (blue), $S_{2}$ (green), and $S_{3}$ (red) for 1 ps following photoexcitation. (b) Relative diabatic state populations of $S_{1}$ (blue), $S_{2}$ (green), $S_{3}$ (red), the triplet $\left(\mathrm{T}_{1-4}\right)$ states (purple), for 1 ps following photoexcitation. The black dashed line is the biexponential fit of the $S_{3}$ diabatic state populations.

after photoexcitation. After only a few femtoseconds IC from $\mathrm{S}_{3}$ to the $S_{2}$ and $S_{1}$ states is observed. The population of these states occurs almost simultaneously due to the strong coupling between $S_{1}$ and $S_{2}$. The electronic energy of the wavepacket on $\mathrm{S}_{3}$ is then converted into kinetic energy on the lower states, resulting in an incoherent distribution of vibrationally hot levels. Initially (up to $200 \mathrm{fs}$ ), the two states $\left(S_{1}\right.$ and $\left.S_{2}\right)$ can be said to be in equilibrium; however, as the dynamics proceeds, the population occurs increasingly in the $S_{1}$ state. Eventually, vibrational cooling and energy transfer to the solvent will completely relax the wavepacket into $S_{1}$; however, this occurs on a longer time scale than presented here. ${ }^{33}$

A more detailed insight into the dynamics may be obtained from the position and width of the wavepacket in each state along the important modes. This is shown in Figure 4 for the totally symmetric $\left(\nu_{8}\right)$ and PJT $\left(\nu_{21}\right)$ modes. In the $S_{3}$ state (Figure $4 \mathrm{a}, \mathrm{d}$ ) the dominant motion is that of the totally symmetric $\nu_{8}$ mode that oscillates around $Q=-0.5$ with a vibrational period of $300 \mathrm{fs}$. This displacement from the FC 


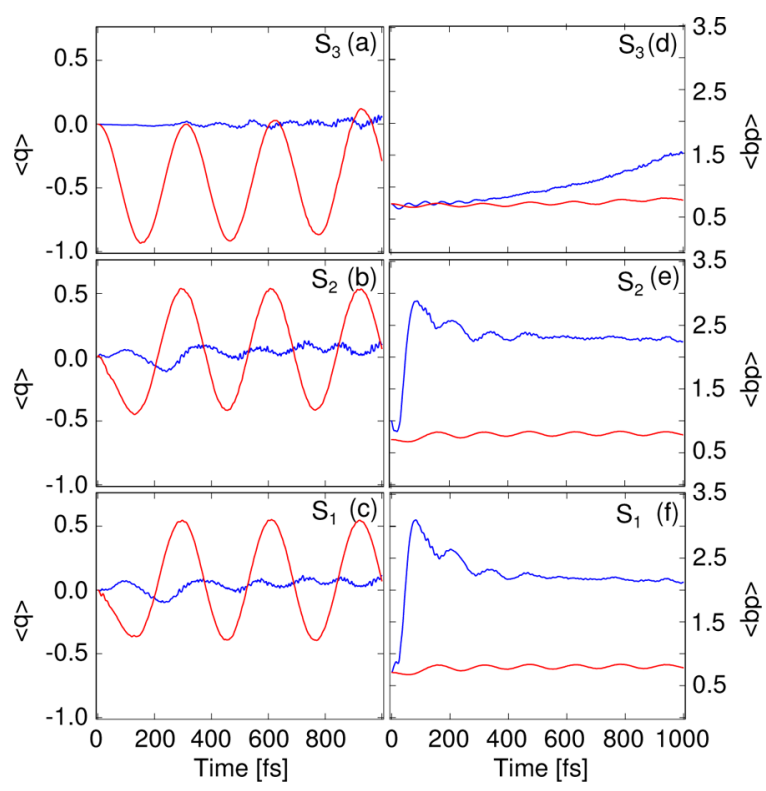

Figure 4. Expectation value of the position, $\langle q\rangle$, and of the width, $\langle d q\rangle$, of the wavepacket in the excited singlet states: (a) and (d) $S_{3}$; (b) and (e) $S_{2}$; (c) and (f) $S_{1}$. Colors: red, $\nu_{8}$; blue, $\nu_{21}$.

point (i.e., $Q=0$ ) corresponds to a slight elongation of the $\mathrm{Cu}-\mathrm{N}$ bond distance, which is the initial motion in the photoexcited dynamics and enables population transfer into the lower states. Along $\nu_{21}$ no significant dynamics are observed in $\mathrm{S}_{3}$ due to the harmonic nature of the potential.

In the $S_{2}$ (Figure $4 b, e$ ) and $S_{1}$ (Figure $4 c, f$ ) states, the wavepacket motions are somewhat similar, due to their strong coupling. We observe, in contrast to the dynamics in $S_{3}$ that the motion along $\nu_{8}$ corresponds to a contraction of the $\mathrm{Cu}-\mathrm{N}$ bond distance; i.e., $\langle q\rangle$ oscillates around values $>0$. Along $\nu_{21}$ the population in neither the $S_{1}$ nor the $S_{2}$ states (Figure $4 b, c$ ) appears to exhibit any significant displacement from the FC geometry. Instead, due to the vibrational hot system, in these states the dynamics are reflected in the width of the wavepacket $\langle d q\rangle$ rather than in its position. Here, at early times we observe a large increase in the width of the wavepacket along $\nu_{21}$ (Figure $4 \mathrm{e}, \mathrm{f}$ ). At later times a gradual decrease in this width is observed, highlighting the beginning of vibrational relaxation.

To compare these simulations to the experimentally observed dynamics, we fit the decay of the $S_{3}$ population with a biexponential. The best fit in Figure $3 \mathrm{a}$ was obtained with two decay times of $\tau_{1} \sim 113$ fs and $\tau_{2} \sim 600$ fs. The first corresponds to the IC from $S_{3}$ to $S_{2}$ and $S_{1}$. While tempting to simply assign the second to the PJT distortion, we found this is not strictly the case. Indeed, the diabatic basis as presented thus far would not show a PJT distortion, and therefore, for the dynamics associated with this component, we must transform it into the adiabatic representation.

Figure 5a shows the adiabatic populations of the $S_{1}^{a}$ (blue), $S_{2}^{a}$ (green), and $S_{3}^{a}$ (red) obtained by a unitary transformation of the diabatic state populations presented in Figure 3. For clarity we have added an ${ }^{a}$ to denote the adiabatic state. Here, $S_{3}^{a}$ shows a decay similar to that observed in the diabatic representation; however, we now observe that, due to the strong coupling between the states, $S_{2}^{a}$ acts as a doorway for population transfer into $S_{1}^{a}$.

To obtain the time scale for the PJT distortion and enable comparison to the experimental time scales, we plot in Figure $5 \mathrm{~b}$ the portion of the wavepacket in a distorted geometry on $S_{1}^{a}$, which is obtained by integrating for $Q<-1.0$ and $Q>1.0$ along the PJT mode (Figure 2 and Figure $5 \mathrm{~b}$ inset). This shows an exponential rise and at $1 \mathrm{ps}, \sim 50 \%$ of the total wavepacket population in the $S_{1}^{a}$ state is in the PJT configuration. The black line in Figure $5 \mathrm{~b}$ shows the fit of this population, which yields a time constant of $400 \mathrm{fs}$. Although faster than the time reported for $\left[\mathrm{Cu}(\mathrm{dmp})_{2}\right]^{+}$in dichloromethane, this is in close agreement with the time constant for the PJT distortion reported in lower viscosity solvents. ${ }^{32}$ This is expected because the present simulations do not incorporate the frictional effects of the environment and, therefore, the time scale extracted represents the lower limit of the experimentally observed time constants.

Intersystem Crossing. Here we repeat the dynamics performed in the previous section but include SOC (Tables S7
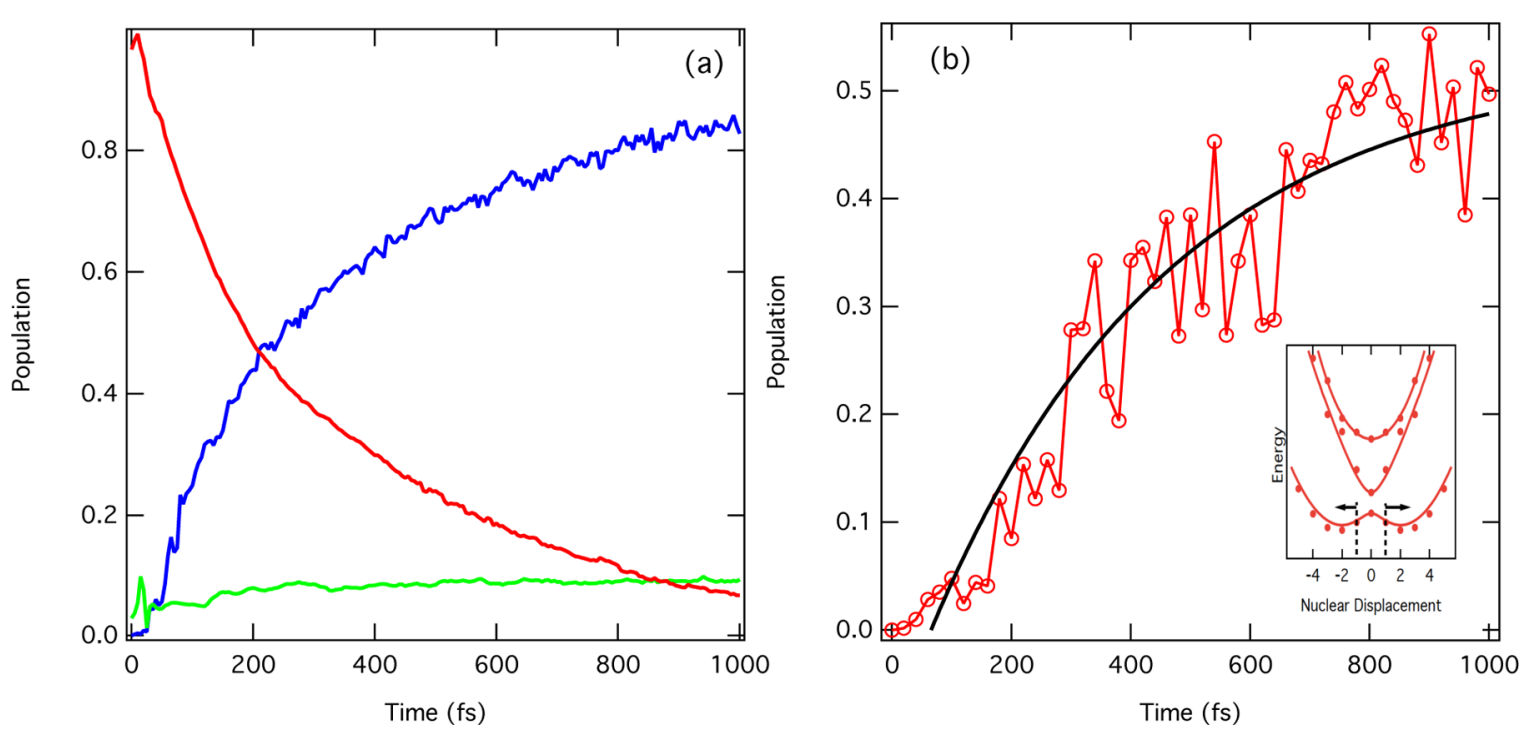

Figure 5. (a) Relative adiabatic population of $S_{1}^{a}$ (blue), $S_{2}^{a}$ (green), and $S_{3}^{a}$ (red) for 1 ps following photoexcitation. (b) Population of the wavepacket in the adiabatic $S_{1}$ in the distorted PJT minimum (red). The black dashed line corresponds to a single exponential fit with a time constant of $400 \mathrm{fs}$. 

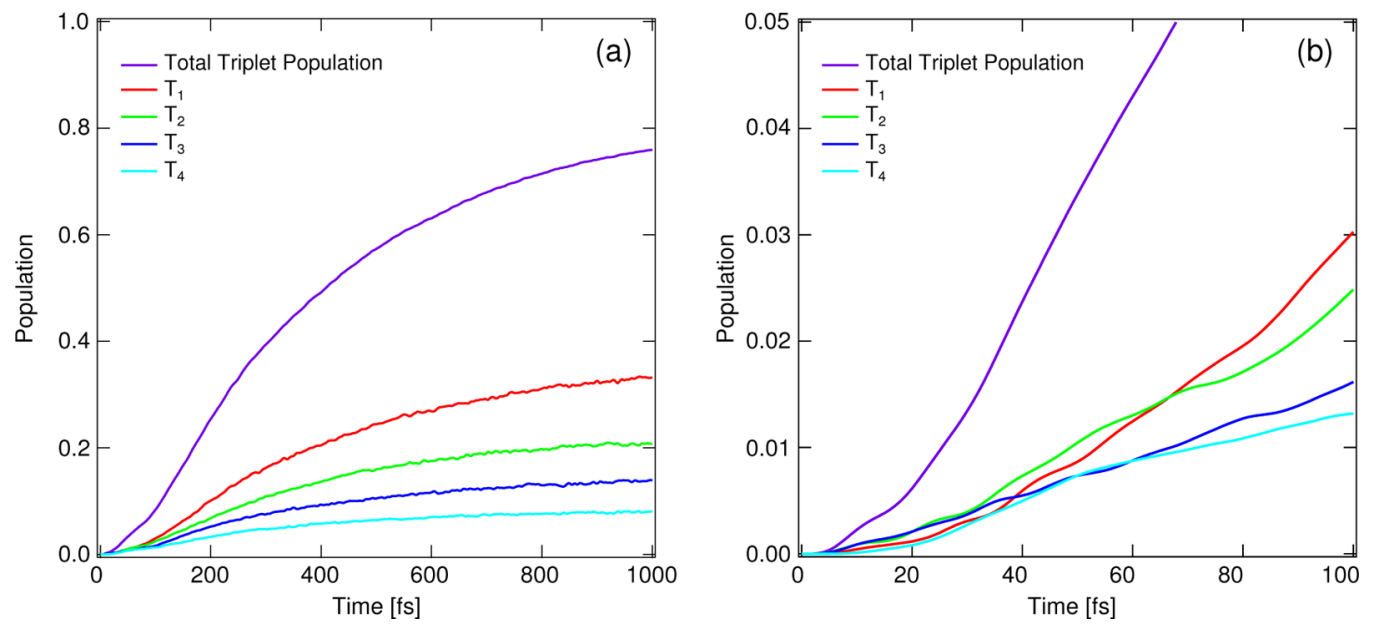

Figure 6. (a) The relative diabatic population of the triplet states $T_{1}$ (red), $T_{2}$ (green), $T_{3}$ (dark blue), and $T_{4}$ (light blue) for 1 ps following photoexcitation. (b) Zoom of the first 100 fs.
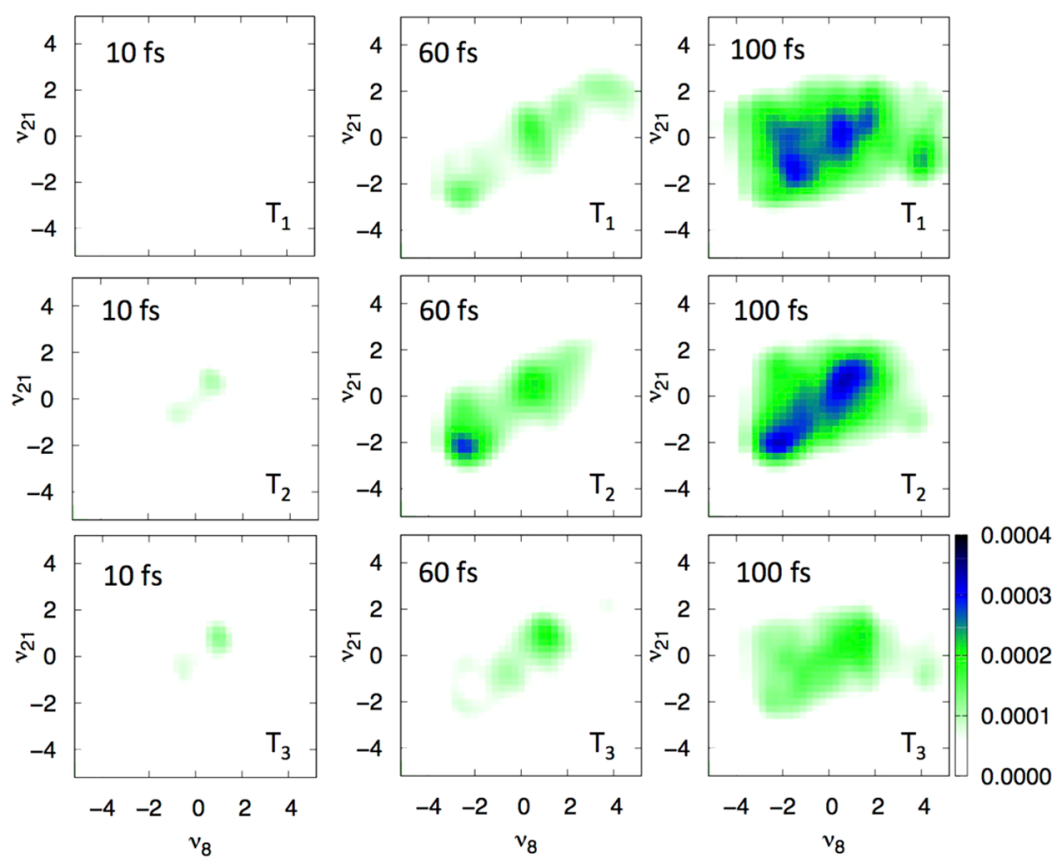

Figure 7. Snapshots of the wavepacket density in the triplet states projected along $\nu_{8}$ and $\nu_{21}$ at time delays of 10,60 , and $100 \mathrm{fs}$ after photoexcitation.

and S8 and Figures S1-S3, Supporting Information), which permits ISC. Figure $3 \mathrm{~b}$ shows the relative diabatic singlet and triplet state populations during the first picosecond. It shows that the decay of the $S_{3}$ population is slightly faster than Figure 3a (i.e., without ISC). However, the largest difference is observed in the population of the $S_{1}$ and $S_{2}$ states which, due to presence of the new triplet relaxation channels, do not exceed a relative population of 0.2 . Significantly, during the first picosecond we observe a total of $80 \%$ of the wavepacket populating the triplet states consistent with ultrafast ISC. This observation disagrees with the conclusions by Tahara et al. ${ }^{32,34}$ but agrees with the original interpretation of Chen and coworkers $^{28}$ and Siddique et al. ${ }^{30}$

The biexponential fit of the $S_{3}$ population decay was again performed and in this case the first decay, $\tau_{1}=103 \mathrm{fs}$, becomes only slightly faster than in the previous section. The slower component is shorter, $\tau_{2}=416 \mathrm{fs}$, because the presence of triplet states and SOC opens new relaxation channels. The time scale for the PJT distortion remains, as found in the previous section, $\sim 400$ fs but, due to the presence of the ISC, exhibits a significantly smaller population.

Figure 6a shows the population of the individual triplet states during the dynamics. Due to the strong vibronic coupling within the triplet manifold and the small energy gap between the states we see a distribution of the population across all of the triplets. This population distribution will remain until, at later times, vibrational cooling relaxes the wavepacket into the flattened geometry of the $\mathrm{T}_{1}$ state. To understand the mechanism of the ISC process, Figure $6 \mathrm{~b}$ zooms into the first $100 \mathrm{fs}$ of the populations kinetics. This shows that ISC initially occurs via $S_{1} \rightarrow T_{2}$ and $S_{1} \rightarrow T_{3}$, due to the strong spin-orbit coupling and degeneracies of these three states along the PJT $\left(\nu_{21}\right)$ mode (Figure 2b) ${ }^{31}$ Indeed, at this point it is important to note that in all of the previous studies the $S_{1} / T_{1}$ ISC was 
considered the only relaxation channel to the lowest triplet state. The present simulations demonstrate that in fact, ISC occurs via a dynamical effect by traversing a region where the coupled singlet and triplet states are degenerate leading to efficient and multiple ultrafast ISC channels.

This is highlighted in Figure 7, which shows snapshots of the wavepacket density in the triplet states projected along $\nu_{8}$ and $\nu_{21}$ at time delays of 10, 60, and $100 \mathrm{fs}$. After $10 \mathrm{fs}$, we observe a very small population of the triplet states in the $T_{2}$ and $T_{3}$ states only. As expected, this occurs at $Q \sim \pm 1$ along $\nu_{21}$, where the $S_{1}$ is degenerate with the $T_{2}$ and $T_{3}$ states. At later times, as observed in Figure 6, the other triplet states become increasingly populated and, due to the strong vibronic coupling and by virtue of it being the lowest state, the majority of the population resides in $T_{1}$. However, for the time scales shown here, as the electronic energy of the population is converted into kinetic energy, giving rise to an incoherent superposition of vibrationally hot levels distributed among the triplet states.

\section{DISCUSSION}

In the previous section we have presented first-principles quantum dynamics simulations which have been used to identify the key mechanistic steps during the excited state nonadiabatic dynamics of $\left[\mathrm{Cu}(\mathrm{dmp})_{2}\right]^{+}$. A summary of the time scales extracted from this work compared to recent experiments is shown in Table 2. As observed, these results compare

Table 2. Time Constants (ps) of the Key Mechanistic Processes Extracted from the Quantum Dynamics Compared to Recent Experimental Conclusions

\begin{tabular}{|c|c|c|c|c|c|}
\hline & $\begin{array}{l}\text { this } \\
\text { work }\end{array}$ & ref 28 & ref 30 & ref 33 & ref 32 \\
\hline $\begin{array}{l}\mathrm{S}_{3} \rightarrow \mathrm{S}_{2} \text { and } \\
\mathrm{S}_{1}\end{array}$ & 0.10 & & & 0.08 & 0.045 \\
\hline$S_{1}^{\mathrm{FC}} \rightarrow S_{1}^{\mathrm{PJT}}$ & 0.40 & $10-20$ & & 0.10 & $0.34-0.70$ \\
\hline $\begin{array}{l}\mathrm{SS}_{1}^{\mathrm{FC}} \rightarrow \\
\quad \text { triplets }^{\mathrm{fc}}\end{array}$ & 0.45 & $0.5-0.7$ & & & \\
\hline $\begin{array}{l}S_{1}^{\mathrm{PJT}} \rightarrow \\
\text { triplets }^{\mathrm{pjt}}\end{array}$ & $a$ & & $13-16$ & $10-15$ & 7.40 \\
\hline
\end{tabular}

${ }^{a}$ Due to the longer time constant of this component, it is not addressed in this work. However, although existence of this component observed in previous experiments is undeniable, our simulations point to an additional relaxation channel and a strong branching of the population kinetics between the $S_{1}^{\mathrm{FC}} \rightarrow S_{1}^{\mathrm{PJT}}$ and $S_{1}^{\mathrm{FC}}$ $\rightarrow \mathrm{T}_{2,3}^{\mathrm{FC}}$ pathways.

favorably to recent experimental measurements, ${ }^{33,32}$ and clarify an important point, which has been widely disputed within the literature. Our simulations demonstrate that $\sim 80 \%$ of the wavepacket crosses into the triplet states within the first picosecond. The majority of this occurs near the FC geometry where the $S_{1}$ is degenerate with both the $T_{2}$ and $T_{3}$. This confirms that ISC occurs on the sub picosecond time scale in $\left[\mathrm{Cu}(\mathrm{dmp})_{2}\right]^{+}$. In this section, we discuss and interpret our results in relation to recent experimental findings, ${ }^{32,33}$ especially concerning the observed ultrafast ISC.

Using either femtosecond emission ${ }^{32}$ or absorption spectroscopy ${ }^{33}$ the fastest components of the dynamics exhibit time constants of $\sim 45$ or $\sim 80 \mathrm{fs}$, respectively. In both cases, this was assigned to the decay of the initially excited $\mathrm{S}_{3}$ state via IC (see (a) in Figure 8). This is in good agreement with the $\sim 100 \mathrm{fs}$ decay component identified in this work, especially considering that the 45 fs component was extracted using an experimental

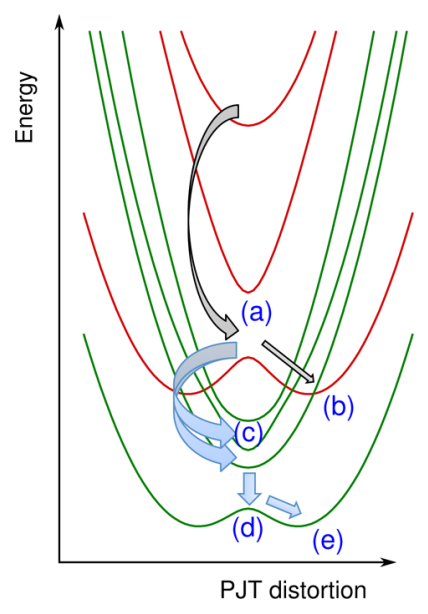

Figure 8. Schematic representation of the key dynamical steps occurring within the ultrafast nonadiabatic dynamics of $\left[\mathrm{Cu}(\mathrm{dmp})_{2}\right]^{+}$ studied here. (a) Internal conversion from $S_{3}$ to $S_{2}$ and $S_{1}: \tau=45$ and $80 \mathrm{fs}^{32,33} 100 \mathrm{fs}$ (this work). (b) Vibrational relaxation from $S_{1}^{\mathrm{FC}}$ to $S_{1}^{\mathrm{PJT}} \tau=340-700 \mathrm{fs}^{32} 400 \mathrm{fs}$ (this work). (c) Ultrafast ISC from $S_{1}^{\mathrm{FC}}$ to $\mathrm{T}_{2}^{\mathrm{FC}}$ and $\mathrm{T}_{3}^{\mathrm{FC}}, \tau=450 \mathrm{fs}$ (this work). (d) IC within the triplets from $\mathrm{T}_{2,3}^{\mathrm{FC}}$ to $\mathrm{T}_{1}^{\mathrm{FC}}$. (e) Vibrational relaxation from $\mathrm{T}_{1}^{\mathrm{FC}}$ to $\mathrm{T}_{1}^{\mathrm{PJT}}$.

setup with a temporal resolution of $200 \mathrm{fs}^{32}$ Our dynamics confirms this assignment of population transfer from $S_{3}$ to the $S_{2}$ and $S_{1}$ states. In particular, the strong coupling between the latter two gives rise to a relaxation cascade and the rapid nature of this decay is due to population transfer near or at the FC region; i.e., very little nuclear dynamics is required. Besides IC, the authors of ref 32 also report that the overall quantum yield of this transition is 0.7 . They concluded that the remaining portion of the wavepacket passes directly from the $S_{3}$ to the $T_{1}$ state on the nanosecond time scale. Although this latter observation is not directly addressed in this work, this conclusion is consistent with our SOC calculations presented in Table S7 (Supporting Information), which show that there is SOC between $S_{3}$ and $T_{1}$ at the FC geometry $\left(\sim 180 \mathrm{~cm}^{-1}\right)$ and also between the $S_{3}$ and $T_{2}\left(\sim 250 \mathrm{~cm}^{-1}\right)$, providing two possible relaxation channels. Though these SOC matrix elements are of similar magnitude to those between the $S_{1}$ and the $\mathrm{T}_{2} / \mathrm{T}_{3}$ states, the ISC occurs on the nanosecond time scale, in this case due to the large energy gap $(\sim 0.5 \mathrm{eV})$ between the coupled states.

Tahara and co-workers ${ }^{32}$ reported that the second dynamical process has a time constant between 340 and $700 \mathrm{fs}$. In contrast to the previous decay component, this time constant exhibits a slight dependence on the viscosity of the solvent and consequently it was assigned to the flattening dynamics associated with the PJT structural distortion. Indeed, the simulations presented here confirms this assignment and at these longer times we observe population in the $S_{1}$ minimum (Figure 5). The time constant extracted from our simulations ( $\sim 400 \mathrm{fs}$ ) represents the lower limit of these experimental time scales as the effect of the friction imparted by the solvent is not incorporated in our present simulations.

As previously stated, while these two previous dynamical components agree closely with recent experimental conclusions, upon inclusion of ISC (Figure 3b), we observe that $\sim 80 \%$ of the population is in the triplets within the first picosecond. This is contrary to the conclusions of refs 27 and 32-34, which have instead reported that ISC only occurs after 10 ps at the flattened PJT geometry. However, the 
interpretations of all of these studies were based on the assumption that SOC is only important between the $S_{1}$ and $T_{1}$ states. Our present simulations, which also include SOC between the other close lying triplet states, points to a strong branching of the population kinetics between the $\mathrm{S}_{1}^{\mathrm{FC}} \rightarrow S_{1}^{\mathrm{PJT}}$ (see (b) in Figure 8) and $S_{1}^{\mathrm{FC}} \rightarrow \mathrm{T}_{2,3}^{\mathrm{FC}}$ (see (c) in Figure 8) pathways. Our simulations show that although both occur, the latter is favored. Importantly, we demonstrate that ISC initially occurs via the $S_{1} \rightarrow T_{2}$ and $T_{3}$ pathways, due to the strong coupling and degeneracies of these states along the PJT $\left(\nu_{21}\right)$ mode. ${ }^{31}$ This strong branching ratio in favor of ultrafast ISC is in close agreement with the conclusions of ref 30, and although this is contrary to the model proposed by Tahara and coworkers, ${ }^{32}$ who assumed a unity quantum yield from $\mathrm{S}_{1}^{\mathrm{FC}} \rightarrow$ $S_{1}^{\text {PJT }}$, they also acknowledged the likelihood of a sizable uncertainty.

\section{CONCLUSIONS}

We have applied the vibronic coupling Hamiltonian and quantum dynamics within the framework of the MCTDH method to study excited state nonadiabatic dynamics of $\left[\mathrm{Cu}(\mathrm{dmp})_{2}\right]^{+}$. Including the dominant nuclear and electronic degrees of freedom we have obtained excited state decay components that agree with those observed experimentally. Our present simulations lead us to the following description of the excited state dynamics. After photoexcitation into the optically bright $S_{3}$ state, the system rapidly decays into $S_{2}$ at the FC geometry. At this point and due to the strong coupling between the almost degenerate $S_{1}$ and $S_{2}$ states, IC into the $S_{1}$ state occurs almost simultaneously. This initial population decay is dominant for the first $\sim 150-200$ fs of the dynamics. Vibrational relaxation into the PJT geometry then occurs with a time constant of $\sim 400$ fs. Experimentally, this process occurs between 340 and $700 \mathrm{fs}$, depending on the viscosity of the solvent. Finally, the strong SOC between singlet and triplet manifolds combined with the degeneracy of the $S_{1}, T_{2}$, and $T_{3}$ states near the FC geometry gives rise to multiple ISC channels, which is the driving force behind the occurrence of ultrafast ISC. This finding of ultrafast ISC has significant implications if one wishes to apply these complexes to solar energy conversion. Indeed, Huang et al. ${ }^{52}$ recently demonstrated that, when a related $\mathrm{Cu}(\mathrm{I})$ - phenanthroline complex is attached to $\mathrm{TiO}_{2}$, charge injection from the ${ }^{1} \mathrm{MLCT}$ is 2 orders of magnitude faster than that from the ${ }^{3} \mathrm{MLCT}$. Consequently, for efficient charge injection one needs to restrict ISC.

\section{ASSOCIATED CONTENT}

\section{S Supporting Information}

Symmetries and frequencies, excitation energies and oscillator strengths, linear coupling constants, Morse parameters, secondorder parameters and spin-orbit couplings can be found in the Supporting Information. This material is available free of charge via the Internet at http://pubs.acs.org.

\section{AUTHOR INFORMATION}

\section{Corresponding Author}

*T. J. Penfold. E-mail: thomas.penfold@psi.ch.

\section{Notes}

The authors declare no competing financial interest.

\section{ACKNOWLEDGMENTS}

We thank the Swiss National Science Foundation (Grant 200021-137717).

\section{REFERENCES}

(1) Kumar, B.; Llorente, M.; Froehlich, J.; Dang, T.; Sathrum, A.; Kubiak, C. P. Photochemical and Photoelectrochemical Reduction of CO 2. Annu. Rev. Phys. Chem. 2012, 63, 541-569.

(2) Gratzel, M. Dye-Sensitized Solar Cells. J. Photochem. Photobiol. C 2003, 4, 145-153.

(3) Holder, E.; Langeveld, B.; Schubert, U. New Trends in the Use of Transition Metal-Ligand Complexes for Applications in Electroluminescent Devices. Adv. Mater. 2005, 17, 1109-1121.

(4) Köppel, H.; Domcke, W.; Cederbaum, L. Multimode Molecular Dynamics Beyond the Born-Oppenheimer Approximation. Adv. Chem. Phys. 1984, 57, 59-246.

(5) Worth, G.; Cederbaum, L. Beyond Born-Oppenheimer: Molecular Dynamics Through a Conical Intersection. Annu. Rev. Phys. Chem. 2004, 55, 127-158.

(6) Forster, L. S. Intersystem Crossing in Transition Metal Complexes. Coord. Chem. Rev. 2006, 250, 2023-2033.

(7) Fantacci, S.; Ronca, E.; De Angelis, F. Impact of Spin-Orbit Coupling on Photocurrent Generation in Ruthenium Dye-Sensitized Solar Cells. J. Phys. Chem. Lett. 2014, 5, 375-380.

(8) Marian, C. M. Spin-Orbit Coupling and Intersystem Crossing in Molecules. Wiley Interdiscip. Rev.: Comput. Mol. Sci. 2011, 2, 187-203.

(9) Damrauer, N. H. Femtosecond Dynamics of Excited-State Evolution in $\left[\mathrm{Ru}(\mathrm{bpy})_{3}\right]_{2}{ }^{+}$. Science 1997, 275, 54-57.

(10) Yeh, A. T. Ultrafast Electron Localization Dynamics Following Photo-Induced Charge Transfer. Science 2000, 289, 935-938.

(11) Bhasikuttan, A. C.; Suzuki, M.; Nakashima, S.; Okada, T. Ultrafast Fluorescence Detection in Tris $\left(2,2^{\prime}\right.$-bipyridine)ruthenium(II) Complex in Solution: Relaxation Dynamics Involving Higher Excited States. J. Am. Chem. Soc. 2002, 124, 8398-8405.

(12) Cannizzo, A.; van Mourik, F.; Gawelda, W.; Zgrablic, G.; Bressler, C.; Chergui, M. Broadband Femtosecond Fluorescence Spectroscopy of $\left[\mathrm{Ru}(\mathrm{bpy})_{3}\right]^{2+}$. Angew. Chem., Int. Ed. 2006, 45, 31743176.

(13) Cannizzo, A.; Blanco-Rodriguez, A. M.; el Nahhas, A.; Sebera, J.; Zalis, S.; Vlcek, A. J.; Chergui, M. Femtosecond Fluorescence and Intersystem Crossing in rhenium(I) carbonyl-bipyridine Complexes. J. Am. Chem. Soc. 2008, 130, 8967-8974.

(14) van der Veen, R. M.; Cannizzo, A.; van Mourik, F.; Vlcek, A. J.; Chergui, M. Vibrational Relaxation and Intersystem Crossing of Binuclear Metal Complexes in Solution. J. Am. Chem. Soc. 2011, 133, 305-315.

(15) Chergui, M. On the Interplay between Charge, Spin and Structural Dynamics in Transition Metal Complexes. Dalton Trans. 2012, 41, 13022-13029.

(16) Siebrand, W. Mechanisms of Intersystem Crossing in Aromatic Hydrocarbons. Chem. Phys. Lett. 1970, 6, 192-194.

(17) Lawetz, V.; Orlandi, G.; Siebrand, W. Theory of Intersystem Crossing in Aromatic Hydrocarbons. J. Chem. Phys. 1972, 56, 4058.

(18) McMillin, D. R.; Kirchhoff, J. R.; Goodwin, K. V. Exciplex Quenching of Photo-excited Copper Complexes. Coord. Chem. Rev. 1985, 64, 83-92.

(19) Armaroli, N. Photoactive Mono- and Polynuclear $\mathrm{Cu}(\mathrm{I})$ Phenanthrolines. A Viable Alternative to $\mathrm{Ru}(\mathrm{II})$-Polypyridines? Chem. Soc. Rev. 2001, 30, 113-124.

(20) Juris, A.; Balzani, V.; Barigelletti, F.; Campagna, S.; Belser, P.; von Zelewsky, A. Ru(II) Polypyridine Complexes: Photophysics, Photochemistry, Eletrochemistry, and Chemiluminescence. Coord. Chem. Rev. 1988, 84, 85-277.

(21) Zgierski, M. Cu(I)-2,9-dimethyl-1,10-phenanthroline: Density Functional Study of the Structure, Vibrational Force Field, and Excited Electronic States. J. Chem. Phys. 2003, 118, 4045. 
(22) Cunningham, C.; Cunningham, K.; Michalec, J.; McMillin, D. Cooperative Substituent Effects on the Excited States of Copper Phenanthrolines. Inorg. Chem. 1999, 38, 4388-4392.

(23) Eggleston, M. K.; McMillin, D. R.; Koenig, K. S.; Pallenberg, A. J. Steric Effects in the Ground and Excited States of $\mathrm{Cu}(\mathrm{NN})_{2}{ }^{+}$ Systems. Inorg. Chem. 1997, 36, 172-176.

(24) Penfold, T. J.; et al. Solvent Induced Luminescence Quenching: Static and Time-Resolved X-Ray Absorption Spectroscopy of a Copper(I) Phenanthroline Complex. J. Phys. Chem. A 2013, 117, 4591-4601.

(25) McCusker, C. E.; Castellano, F. N. Design of a Long-Lifetime, Earth-Abundant, Aqueous Compatible $\mathrm{Cu}$ (I) Photosensitizer Using Cooperative Steric Effects. Inorg. Chem. 2013, 52, 8114-8120.

(26) Khnayzer, R. S.; McCusker, C. E.; Olaiya, B. S.; Castellano, F. N. Robust Cuprous Phenanthroline Sensitizer for Solar Hydrogen Photocatalysis. J. Am. Chem. Soc. 2013, 135, 14068-14070.

(27) Iwamura, M.; Takeuchi, S.; Tahara, T. Substituent Effect on the Photoinduced Structural Change of $\mathrm{Cu}(\mathrm{I})$ Complexes Observed by Femtosecond Emission Spectroscopy. Phys. Chem. Chem. Phys. 2014, $16,4143-4154$.

(28) Chen, L.; Shaw, G.; Novozhilova, I.; Liu, T.; Jennings, G.; Attenkofer, K.; Meyer, G.; Coppens, P. MLCT State Structure and Dynamics of a Copper(I) diimine Complex Characterized by Pumpprobe X-ray and Laser Spectroscopies and DFT calculations. J. Am. Chem. Soc. 2003, 125, 7022-7034.

(29) Note that $S_{3}$ in this present paper is sometimes referred to as $S_{2}$ in other works. This is because at $D_{2 d}$ symmetry $S_{1}$ and $S_{2}$ are degenerate. However, we find that the lowest energy structure has a $D_{2}$ symmetry, which breaks the degeneracy.

(30) Siddique, Z.; Yamamoto, Y.; Ohno, T.; Nozaki, K. Structuredependent Photophysical Properties of Singlet and Triplet Metal-toligand Charge Transfer States in Copper(I) bis(diimine) Compounds. Inorg. Chem. 2003, 42, 6366-6378.

(31) Capano, G.; Penfold, T. J.; Rothlisberger, U.; Tavernelli, I. A Vibronic Coupling Hamiltonian to Describe the Ultrafast Excited State Dynamics of a $\mathrm{Cu}(\mathrm{I})$-Phenanthroline Complex. Chimia 2014, 68, 227-230.

(32) Iwamura, M.; Takeuchi, S.; Tahara, T. Real-time Observation of the Photoinduced Structural Change of Bis(2,9-dimethyl-1,10phenanthroline)copper(I) by Femtosecond Fluorescence Spectroscopy: A Realistic Potential Curve of the Jahn-Teller Distortion. J. Am. Chem. Soc. 2007, 129, 5248-5256.

(33) Shaw, G. B.; Grant, C. D.; Shirota, H.; Castner, E. W.; Meyer, G. J.; Chen, L. X. Ultrafast Structural Rearrangements in the MLCT Excited State for Copper(I) Bis-phenanthrolines in Solution. J. Am. Chem. Soc. 2007, 129, 2147-2160.

(34) Iwamura, M.; Watanabe, H.; Ishii, K.; Takeuchi, S.; Tahara, T. Coherent Nuclear Dynamics in Ultrafast Photoinduced Structural Change of Bis(diimine)copper(I) Complex. J. Am. Chem. Soc. 2011, $133,7728-7736$.

(35) Penfold, T.; Spesyvtsev, R.; Kirkby, O. M.; Minns, R.; Parker, D.; Fielding, H.; Worth, G. Quantum Dynamics Study of the Competing Ultrafast Intersystem Crossing and Internal Conversion in the "Channel 3" Region of Benzene. J. Chem. Phys. 2012, 137, 204310.

(36) Beck, M. H.; Jäckle, A.; Worth, G. A.; Meyer, H.-D. The Multiconfiguration Time-Dependent Hartree Method: A Highly Efficient Algorithm for Propagating Wavepackets. Phys. Rep. 2000, 324, 1-105.

(37) Köppel, H.; Domcke, W.; Cederbaum, L. S. Multimode Molecular Dynamics Beyond the Born-Oppenheimer Approximation 1984, 57, 59-246.

(38) Penfold, T.; Worth, G. A Model Hamiltonian to Simulate the Complex Photochemistry of Benzene II. J. Chem. Phys. 2009, 131, 190-199.

(39) Frisch, M. J.; et al. Gaussian 09, Revision A.1; Gaussian Inc.: Wallingford, CT, 2009.

(40) Zhao, Y.; Truhlar, D. G. The M06 Suite of Density Functionals for Main Group Thermochemistry, Thermochemical Kinetics, Noncovalent Interactions, Excited States, and Transition Elements: Two
New Functionals and Systematic Testing of Four M06-Class Functionals and 12 other Functionals. Theor. Chem. Acc. 2007, 120, 215-241.

(41) Stephens, P. J.; Devlin, F. J.; Chabalowski, C. F.; Frisch, M. J. Ab Initio Calculation of Vibrational Absorption and Circular Dichroism Spectra Using Density Functional Force Fields. J. Phys. Chem. 1994, 98, 11623-11627.

(42) Becke, A. D. Density Functional Thermochemistry. III. The Role of Exact Exchange. J. Chem. Phys. 1993, 98.

(43) Vosko, S. H.; Wilk, L.; Nusair, M. Accurate Spin Dependent Electron Liquid Correlation Energies for Local Spin Density Calculations: A Critical Analysis. Can. J. Phys. 1980, 58, 1200-1211.

(44) Lee, C.; Yang, W.; Parr, R. G. Development of the Colle-Salvetti Correlation Energy Formula into a Functional of the Electron Density. Phys. Rev. B 1988, 37, 785-789.

(45) Wang, F.; Ziegler, T. A Simplified Relativistic Time Dsependent Density Functional Theory Formalism for the Calculations of Excitation Energies Including Spin-Orbit Coupling Effect. J. Chem. Phys. 2005, 123, 154102.

(46) Fonseca Guerra, C.; Snijders, J. G.; Te Velde, G.; Baerends, E. J. Towards an order- N DFT method. Theor. Chem. Acc. 1998, 99, 391403.

(47) van Gisbergen, S.; Snijders, J.; Baerends, E. Implementation of Time-Dependent Density Functional Response Equations. Comput. Phys. Commun. 1999, 118, 119-138.

(48) ADF2009.01; Scientific Computation and Modelling: Amsterdam, 2010; http://www.scm.com/.

(49) Lenthe, E. v.; Baerends, E. J.; Snijders, J. G. Relativistic Regular Two Component Hamiltonians. J. Chem. Phys. 1993, 99.

(50) van Lenthe, E.; Baerends, E. J.; Snijders, J. G. Relativistic Total Energy Using Regular Approximations. J. Chem. Phys. 1994, 101.

(51) Meyer, H.-D.; Manthe, U.; Cederbaum, L. S. The MultiConfigurational Time-Dependent Hartree Approach. Chem. Phys. Lett. 1990, 165, 73-78.

(52) Huang, J.; Buyukcakir, O.; Mara, M. W.; Coskun, A.; Dimitrijevic, N. M.; Barin, G.; Kokhan, O.; Stickrath, A. B.; Ruppert, R; Tiede, D. M.; et al. Highly Efficient Ultrafast Electron Injection from the Singlet MLCT Excited State of Copper(I) Diimine Complexes to $\mathrm{TiO}_{2}$ Nanoparticles. Angew. Chem., Int. Ed. 2012, 51, 12711-12715. 